\title{
Best anthropometric predictors of fasting blood sugar, prediabetes, and diabetes
}

\author{
Idris Muhammad Yakubu*, Yazid Suleiman Kaoje, Tahoma Jabbe and Aliyu Abdullahi Abubakar \\ CBN Diagnostic and Treatment Center, Garki 2, Abuja, Nigeria
}

\begin{abstract}
Background: The association between obesity and type 2 diabetes is well-known. The measure of obesity that best predicts fasting blood sugar levels remains a subject of debate. The objective of the study was to determine the best anthropometric predictor of FBS, prediabetes, and diabetes among apparently healthy bank workers in Nigeria.

Methods: This study analyzed dataset of a cross-sectional survey of apparently healthy 1191 bank workers in 18 state capitals across the six geopolitical regions of Nigeria. The dataset was processed and analyzed with SPSS. Correlation and discriminant analysis were performed to determine the best anthropometric predictors of FBS, prediabetes, and diabetes.

Results: The 1191 subjects were aged $22-59$ years old, $80.2 \%$ were males, and $19.8 \%$ females. The average FBS was $5.2 \pm 1.2 \mathrm{mmol} / 1, \mathrm{BMI} 26.7 \pm 4.6 \mathrm{~kg} / \mathrm{m}^{2}, \mathrm{WC}$ $80.1 \pm 25.1 \mathrm{~cm}, \mathrm{HC} 88.9 \pm 26.7 \mathrm{~cm}$, WHR $0.9 \pm 0.09$, and WHtR $0.47 \pm 0.15$. The prevalence of diabetes was $4 \%$, prediabetes $7 \%$, overweight $42 \%$, and obesity $23 \%$. The best anthropometric predictor of FBS was WC $(r=0.231$, $\mathrm{p}<0.0001$ ), while the best predictor of diabetes was WHR (AUC $0.64,95 \%$ CI $0.55-0.73$ ) and HC for prediabetes (AUC $0.64,95 \%$ CI 0.58-0.69). The best predictors of all the three outcomes combined were WC and WHtR at cut-off values of $87 \mathrm{~cm}$ and 0.5 in both sexes.
\end{abstract}

Conclusions: Both WC and WHtR at cut-off values of $87 \mathrm{~cm}$ and 0.5 are appropriate as discriminative anthropometric indices for both prediabetes and diabetes in both sexes. The finding of $\mathrm{HC}$ as the best predictor of prediabetes calls for further research in this direction.

\begin{abstract}
Abbreviations: BMI: Body mass index; FBS: Fasting blood sugar; HC: Hip circumference; WC: Waist circumference; WHR: Waist hip ratio; WHtR: Waist height ratio.
\end{abstract}

\section{Introduction}

Obesity, now recognized as a chronic non-communicable disease, represents an excessive amount of fat in the body [1]. It is a component of metabolic syndrome and a risk factor for type 2 diabetes, dyslipidemia, hypertension, and cardiovascular disease [2]. The distribution of fat in obesity is a predictor of cardiometabolic risk with visceral obesity carrying more risks than central or general obesity [3].

BMI is the most widely used clinical tool for measuring obesity because it is easy to measure and interpret. However, it does not localize fat distribution or differentiate muscle mass from excess fat [4]. Consequently, other simple anthropometric measures of obesity such as WC, HC, WHR, and WHtR are often used in conjunction with BMI in low-resourced settings where advanced and sophisticated technology for fat assessment is not readily available. Within such clinical settings, WC, WHR, and WHtR are favored as better measures of central and visceral obesity than BMI $[4,5]$.

Visceral and abdominal fat accumulation manifest as central obesity. Central obesity is associated with insulin resistance, type 2 diabetes, metabolic syndrome, and cardiovascular disease [2-5]. Globally, many studies have explored the relationship between the various anthropometric indices and abnormal blood sugar states across divergent geographical, ethnic, socio-economic, sex, and clinical backgrounds with inconsistent results [4-6]. A recent population-based cross-sectional study with 1,000 participants in Babol, northern Iran reported that WC was the best anthropometric predictor of diabetes using AUC when compared with BMI, WHR, and WHtR [4].

There is a paucity of similar studies in sub-Saharan African [7]. Only a few studies explored this phenomenon in Nigeria [8-11]. Most of these studies looked at associations (rather than discriminative and predictive abilities) between some of the anthropometric parameters and outcomes like cardiometabolic risk, FBS, insulin resistance, prediabetes, and diabetes. Few attempts at exploring the best anthropometric predictors of these outcomes returned varying results and no study simultaneously explored FBS, prediabetes, and diabetes.

This study attempted to extend the existing body of knowledge by determining the best anthropometric predictor of FBS, prediabetes, and diabetes using area under the curve (AUC) in receiver operating characteristic (ROC) in nationwide data involving apparently healthy bank employees in Nigeria. Workers in the banking industry live mostly sedantary lifestyles and the prevalence of obesity and perhaps type 2 diabetes are on the increase [12]. There was no similar study on bank employees in Nigeria. This study used simple correlation coefficient to

${ }^{\star}$ Correspondence to: Idris Muhammad Yakubu MBBS, PGDip, MPH, PhD student in Public Health at Texila American University, CBN Diagnostic and Treatment Center, Garki 2, Abuja, Nigeria, E-mail: yidris2000@yahoo.co.uk

Key words: anthropometric indices, fasting blood sugar, prediabetes, diabetes, bank employees

Received: June 13, 2020; Accepted: June 23, 2020; Published: June 29, 2020 
determine the best anthropometric predictor of FBS and discriminative analysis with eyeballing in place of Youden index for determining optimal cut-off values of predictive indices in prediabetes and diabetes.

\section{Materials and methods}

This study was a secondary research which analyzed dataset in medical records collected in 2016, involving apparently healthy bank employees in 18 state capitals across Nigeria's six geopolitical zones. Three branches of a particular bank were selected from each zone based on the availability of complete data. Personal identifiers such as names, dates of birth, identity numbers, department, status, and location were deleted from the primary dataset.

\section{Primary data collection process}

The inclusion criteria for the participants in the primary data collection were last meal at or before $10 \mathrm{pm}$ of the previous night, absence of pregnancy, and absence of symptoms. Apparently healthy subjects with underlying chronic illnesses were not excluded.

The participants' personal information including age and sex were noted in a schedule, followed by anthropometric measurements of weight, height, WC, and HC.

Trained and experienced nurses and doctors (including the authors) collected the primary data using calibrated and quality-assured weight scales (Omron), height scales (Seca), and glucometers (Accu-Chek Roche). Standard procedures were applied in measuring weight, height, WC, HC, and FBS. Weight was measured in kilogram (kg), height, WC, and $\mathrm{HC}$ in centimeter $(\mathrm{cm})$, and FBS in $\mathrm{mmol} / \mathrm{l}$. BMI was defined as weight $(\mathrm{kg})$ divided by height squared $\left(\mathrm{m}^{2}\right)$, WHR as WC divided by $\mathrm{HC}$, and WHtR as WC divided by height. BMI, WHR, WHtR were calculated manually using calculators and Microsoft Excel templates. All measurements were crosschecked to ensure accuracy.

Data processing and editing: The primary data in Excel was imported to SPSS. Only states with complete dataset without missing values were included in this secondary study. Personal identifiers were deleted for ethical reasons. BMI, WHR, and WHtR were cross-checked manually to ensure accuracy.

Statistical analysis: Statistical Package for Social Sciences (SPSS) Version 23 was used for the analysis. FBS level and BMI were grouped according to the World Health Organization (WHO) classifications of obesity and abnormal blood sugar to enable the estimation of prevalence rates. FBS was defined as normal $(<6.1 \mathrm{mmol} / \mathrm{l})$, prediabetes $(6.1-6.9$ $\mathrm{mmol} / \mathrm{l})$ and diabetes $(\geq 7 \mathrm{mmol} / \mathrm{l})$. Underweight was defined as BMI $<$ 18.5 , normal weight $18.5-24.9$, overweight $25-29.9$, and obesity $>30$. The independent variables were age, sex, and anthropometric indices. The dependent variables were FBS levels, prediabetes, and diabetes.

Numeric variables were tested for normal distribution. Prevalence of prediabetes and diabetes was compared based on age, sex, and WHO obesity classes using cross-tabulations. Mean anthropometric indices were compared in both sexes in the prediabetes and diabetes groups using independent samples t-test.

Correlation test and ROC analysis were performed to determine statistically significant associations and discriminating ability, respectively, at p-value $<0.05$ and confidence interval (CI) of $95 \%$. The correlation coefficient, a measure of the strength of association, was used to determine the best anthropometric predictor of FBS. In contrast, AUC was used to ascertain the best anthropometric predictor of prediabetes and diabetes. Eyeballing was used to determine the optimal cut-off values of the anthropometric indices with the best sensitivities and specificities.

\section{Results}

The study had 1191 participants, $80 \%$ of which were men and $20 \%$ women. The age of the participants ranged between 22 and 59 years with a mean of $41.18 \pm 8.58$ years. The mean age of men $(41.33 \pm 8.45)$ was higher than that of women $(40.66 \pm 9.07)$. The overall mean FBS was 5.2 $\pm 1.2 \mathrm{mmol} / \mathrm{l}$, BMI $26.7 \pm 4.6 \mathrm{~kg} / \mathrm{m}^{2}$, WC $80.1 \pm 25.1 \mathrm{~cm}, \mathrm{HC} 88.9 \pm 26.7$ $\mathrm{cm}$, WHR $0.9 \pm 0.09$, and WHtR $0.47 \pm 0.15$. The mean anthropometric measures were higher in women than in men with the exception of WHR which was higher in men (Table 1). The mean FBS was higher in men than in women. FBS, BMI, and WHR had respectively comparable mean, median, and mode with normality plots within acceptable limits of a normal distribution.

The overall prevalence of diabetes was $3.9 \%$, prediabetes $7.1 \%$, overweight $41.9 \%$, and obesity $23.3 \%$. The prevalence of prediabetes and diabetes was higher in men (3.9\% vs $7.5 \%)$ than in women $(3.8 \%$ vs $7 \%$ ) and increased with the extent of obesity (Table 2 ). The prevalence of diabetes increased with age. Prediabetes had a higher prevalence in youths than in middle-aged participants (8.5\% against $7.3 \%)$.

The mean values of all anthropometric indices in both sexes were higher in the prediabetes group than in the diabetes group and lowest in the normal group with the exception of WHR, which remained consistently highest in the diabetes group (Table 3 ).

All of the predictor variables had significant positive correlation with FBS in both sexes except BMI and WHR in women only where the positive correlation was not statistically significant (Table 4). Men had higher correlation coefficients and levels of significance than women. Using the correlation coefficient as a measure of the strength of association, the anthropometric parameter with the strongest association in both sexes was WC $(r=0.231, p<0.0001)$, followed by WHtR $(\mathrm{r}=0.224, \mathrm{p}<0.0001)$, HC $(\mathrm{r}=0.221, \mathrm{p}<0.0001)$, and BMI $(\mathrm{r}=0.097, \mathrm{p}<0.001)$. WHR had the weakest strength with a coefficient of 0.065 and a $\mathrm{p}$-value of 0.024 . Therefore, WC was a better predictor of FBS than WHtR, HC, BMI, or WHR in this order. However, in women HC was slightly better than WHtR (Table 4).

WHR was the best predictor of diabetes in both sexes with the predictive ability higher in women than in men with AUC of 0.70 and 0.64 , respectively. In contrast, the other anthropometric indices demonstrated greater predictive ability in men than in women (Table5 and Figure 1). Women had higher cut-off values and lower sensitivities than men. All sensitivities were less than $70 \%$ in women and at least $70 \%$ in men, except for WHR which had a similar cuff-off value of 0.9 in both sexes and sensitivity of $78 \%$ in women and $73 \%$ in men. WC was the least predictor of diabetes in both men and women.

The anthropometric prediction of prediabetes exhibited interesting and consistent patterns. HC, WC, and WHtR in both sexes had a comparable and greatest discriminating ability, while BMI and WHR had the least. The AUCs and sensitivities were higher in men than in women, while the cut-off values and specificities were greater in women than in men except for WHR (Table 5).

In summary, the best anthropometric predictors of FBS were WC and WHtR in men and WC and HC in women. The best predictors of diabetes were WHR and WC in men, and WHR and BMI in women. In contrast, the best anthropometric predictors of prediabetes were $\mathrm{HC}$ and WC in both sexes. 
Table 1. Univariate statistics of continuous variables

\begin{tabular}{|c|c|c|c|c|c|c|c|}
\hline Measures & Age (years) & FBS & BMI & WC & $\mathrm{HC}$ & WHR & WHtR \\
\hline \multicolumn{8}{|c|}{ Men } \\
\hline Mean & 41.33 & 5.18 & 26.18 & 79.21 & 86.86 & 0.91 & 0.46 \\
\hline Median & 40.00 & 5.10 & 26.00 & 87.00 & 97.00 & 0.90 & 0.50 \\
\hline Mode & 35 & 5.20 & 25.00 & 93.00 & 102.0 & 0.90 & 0.49 \\
\hline Std. Deviation & 8.45 & 1.25 & 4.30 & 25.21 & 26.72 & 0.08 & 0.15 \\
\hline Minimum & 22 & 2.10 & 17.00 & 26.00 & 31.00 & 0.66 & 0.01 \\
\hline Maximum & 59 & 20.40 & 48.00 & 192.0 & 133.0 & 1.88 & 1.13 \\
\hline \multicolumn{8}{|c|}{ Women } \\
\hline Mean & 40.66 & 5.12 & 28.72 & 83.57 & 97.01 & 0.86 & 0.51 \\
\hline Median & 39.00 & 5.00 & 28.00 & 89.00 & 104.00 & 0.87 & 0.54 \\
\hline Mode & 39 & 4.80 & 26.00 & 88.00 & 104.00 & 0.88 & 0.56 \\
\hline Std. Deviation & 9.07 & 1.22 & 5.39 & 24.16 & 25.35 & 0.11 & 0.15 \\
\hline Minimum & 22 & 2.70 & 16.00 & 24.00 & 33.00 & 0.34 & 0.15 \\
\hline Maximum & 59 & 16.60 & 46.00 & 175.00 & 134.00 & 1.84 & 1.03 \\
\hline \multicolumn{8}{|c|}{ Total (combined men and women) } \\
\hline Mean & 41.18 & 5.17 & 26.68 & 80.08 & 88.87 & 0.9 & 0.47 \\
\hline Median & 39.44 & 5.06 & 26 & 88 & 98 & 0.9 & 0.51 \\
\hline Mode & 46 & 5 & 25 & 90 & 102 & 0.9 & 0.5 \\
\hline Std. Deviation & 8.58 & 1.25 & 4.65 & 25.05 & 26.75 & 0.09 & 0.15 \\
\hline Minimum & 22 & 2.1 & 16 & 24 & 31 & 0.34 & 0.01 \\
\hline Maximum & 59 & 20.4 & 48 & 192 & 134 & 1.88 & 1.13 \\
\hline
\end{tabular}

Table 2. The prevalence of prediabetes and diabetes based on age, sex, and BMI (FBS in mmol/l)

\begin{tabular}{|c|c|c|c|c|}
\hline \multirow{2}{*}{ Predictor } & \multicolumn{3}{|c|}{ Prevalence according FBS status } & \multirow{2}{*}{$\mathbf{P}$} \\
\hline & Diabetes & Prediabetes & Normal & \\
\hline \multicolumn{5}{|c|}{ Sex } \\
\hline Male & $37(3.9 \%)$ & $72(7.5 \%)$ & $846(88.6 \%)$ & 0.55 \\
\hline Female & $9(3.8 \%)$ & $13(7.0 \%)$ & $214(90.7 \%)$ & \\
\hline \multicolumn{5}{|c|}{ Age (Years) } \\
\hline $18-39$ & $9(1.4 \%)$ & $44(8.5 \%)$ & $573(91.5 \%)$ & $<0.001$ \\
\hline $40-59$ & $37(6.6 \%)$ & $41(7.3 \%)$ & $480(86.0 \%)$ & \\
\hline \multicolumn{4}{|c|}{ BMI } & \\
\hline Underweight & - & - & $16(100 \%)$ & 0.16 \\
\hline Normal weight & $11(2.8 \%)$ & $22(5.5 \%)$ & $366(91.7 \%)$ & \\
\hline Overweight & $21(4.2 \%)$ & $36(7.2 \%)$ & $442(88.6 \%)$ & \\
\hline Obesity & $14(5.1 \%)$ & $27(9.7 \%)$ & $236(85.2 \%)$ & \\
\hline
\end{tabular}

Table 3. The mean $( \pm \mathrm{SD})$ of anthropometric indices according to the FBS status and sex

\begin{tabular}{|c|c|c|c|}
\hline \multirow{2}{*}{ Anthropometric indices } & \multicolumn{3}{|c|}{ Mean $( \pm \mathrm{SD})$ anthropometric indices according to FBS status in $\mathrm{mmol} / \mathrm{l}$} \\
\hline & Diabetes $(\geq 7)$ & Prediabetes (6.1-6.9) & Normal $(<6.1)$ \\
\hline \multicolumn{4}{|c|}{ Male } \\
\hline BMI & $27.61 \pm 3.78$ & $27.61 \pm 4.87$ & $25.99 \pm 4.238$ \\
\hline WC & $84.54 \pm 26.75$ & $89.33 \pm 20.55$ & $78.12 \pm 25.30$ \\
\hline $\mathrm{HC}$ & $90.22 \pm 28.27$ & $97.39 \pm 20.20$ & $85.82 \pm 26.96$ \\
\hline WHR & $0.94 \pm 0.06$ & $0.92 \pm 0.06$ & $0.91 \pm 0.08$ \\
\hline WHtR & $0.49 \pm 0.16$ & $0.52 \pm 0.12$ & $0.45 \pm 0.15$ \\
\hline \multicolumn{4}{|c|}{ Females } \\
\hline BMI & $29.67 \pm 4.77$ & $30.55 \pm 4.95$ & $28.57 \pm 5.444$ \\
\hline WC & $83.44 \pm 25.79$ & $95.62 \pm 10.44$ & $82.84 \pm 24.56$ \\
\hline $\mathrm{HC}$ & $92.00 \pm 27.32$ & $111.38 \pm 10.46$ & $96.35 \pm 25.70$ \\
\hline WHR & $0.91 \pm 0.06$ & $0.86 \pm 0.09$ & $0.86 \pm 0.11$ \\
\hline WHtR & $0.52 \pm 0.16$ & $0.58 \pm 0.07$ & $0.50 \pm 0.15$ \\
\hline \multicolumn{4}{|c|}{ Total (combined males and females) } \\
\hline BMI & $28.01 \pm 4.02$ & $28.06 \pm 4.970$ & $26.51 \pm 4.62$ \\
\hline WC & $84.33 \pm 26.28$ & $90.29 \pm 19.44$ & $79.07 \pm 25.22$ \\
\hline $\mathrm{HC}$ & $90.57 \pm 27.80$ & $99.53 \pm 19.65$ & $87.95 \pm 27.03$ \\
\hline WHR & $0.93 \pm 0.06$ & $0.91 \pm 0.07$ & $0.90 \pm 0.09$ \\
\hline WHtR & $0.49 \pm 0.16$ & $0.53 \pm 0.12$ & $0.46 \pm 0.15$ \\
\hline
\end{tabular}


Table 4. Correlations between FBS and age, WC, HC, BMI, and WHR

\begin{tabular}{|c|c|c|c|c|c|c|}
\hline Pearson Correlations & Age (years) & WC & WHtR & $\mathrm{HC}$ & BMI & WHR \\
\hline \multicolumn{7}{|c|}{ Men } \\
\hline Correlation coefficient (r) & 0.185 & .247 & .241 & .236 & 0.105 & .075 \\
\hline P-value & $<0.0001$ & $<0.0001$ & $<0.0001$ & $<0.0001$ & 0.001 & 0.021 \\
\hline \multicolumn{7}{|c|}{ Women } \\
\hline Correlation coefficient $(\mathrm{r})$ & 0.167 & 0.170 & 0.175 & 0.180 & 0.101 & 0.032 \\
\hline P-value & 0.01 & 0.009 & 0.007 & 0.005 & 0.121 & 0.621 \\
\hline \multicolumn{7}{|c|}{ Both } \\
\hline Correlation coefficient (r) & 0.183 & 0.231 & 0.224 & 0.221 & 0.097 & 0.065 \\
\hline P-value & $<0.0001$ & $<0.0001$ & $<0.0001$ & $<0.0001$ & 0.001 & 0.024 \\
\hline
\end{tabular}

Table 5. Diagnostic accuracy of anthropometric indices for the prediction of diabetes/prediabetes and optimal cut-off values

\begin{tabular}{|c|c|c|c|c|}
\hline \multicolumn{5}{|c|}{ Diabetes } \\
\hline Anthropometric indices & AUC (95\% CI) & Optimal cut-off & Sensitivity & Specificity \\
\hline \multicolumn{5}{|c|}{ Male } \\
\hline WHR & $0.64(0.55-0.73)$ & 0.90 & $73 \%$ & $44 \%$ \\
\hline WC & $0.62(0.52-0.72)$ & 86.5 & $73 \%$ & $49 \%$ \\
\hline BMI & $0.62(0.54-0.71)$ & 25.5 & $73 \%$ & $50 \%$ \\
\hline WHtR & $0.59(0.49-0.69)$ & 0.49 & $70 \%$ & $43 \%$ \\
\hline $\mathrm{HC}$ & $0.59(0.48-0.69)$ & 94.5 & $70 \%$ & $42 \%$ \\
\hline \multicolumn{5}{|c|}{ Females } \\
\hline WHR & $0.70(0.54-0.86)$ & 0.90 & $78 \%$ & $66 \%$ \\
\hline BMI & $0.56(0.38-0.74)$ & 27.5 & $67 \%$ & $45 \%$ \\
\hline WHtR & $0.54(0.33-0.75)$ & 0.52 & $67 \%$ & $40 \%$ \\
\hline WC & $0.52(0.33-0.72)$ & 86.5 & $67 \%$ & $42 \%$ \\
\hline $\mathrm{HC}$ & $0.44(0.27-0.61)$ & 99.5 & $67 \%$ & $39 \%$ \\
\hline \multicolumn{5}{|c|}{ Total (combined males and females) } \\
\hline WHR & $0.65(0.57-0.72)$ & 0.89 & $74 \%$ & $48 \%$ \\
\hline WC & $0.61(0.51-0.69)$ & 85.5 & $72 \%$ & $45 \%$ \\
\hline BMI & $0.61(0.53-0.68)$ & 25.3 & $74 \%$ & $45 \%$ \\
\hline WHtR & $0.58(0.49-0.67)$ & 0.49 & $70 \%$ & $43 \%$ \\
\hline $\mathrm{HC}$ & $0.56(0.47-0.64)$ & 95.5 & $70 \%$ & $41 \%$ \\
\hline \multicolumn{5}{|c|}{ Prediabetes } \\
\hline Anthropometric indices & $\mathrm{AUC}(95 \% \mathrm{CI})$ & Optimal cut-off & Sensitivity & Specificity \\
\hline \multicolumn{5}{|c|}{ Male } \\
\hline $\mathrm{HC}$ & $0.64(0.59-0.70)$ & 96.5 & $73 \%$ & $49 \%$ \\
\hline WC & $0.63(0.57-0.69)$ & 85.5 & $72 \%$ & $48 \%$ \\
\hline WHtR & $0.63(0.57-0.69)$ & 0.49 & $75 \%$ & $44 \%$ \\
\hline BMI & $0.60(0.54-0.67)$ & 25.5 & $65 \%$ & $50 \%$ \\
\hline WHR & $0.53(0.46-0.60)$ & 0.90 & $57 \%$ & $43 \%$ \\
\hline \multicolumn{5}{|c|}{ Females } \\
\hline $\mathrm{HC}$ & $0.65(0.52-0.78)$ & 107.5 & $67 \%$ & $62 \%$ \\
\hline WC & $0.61(0.48-0.73)$ & 88.5 & $67 \%$ & $48 \%$ \\
\hline WHtR & $0.61(0.48-0.74)$ & 0.55 & $60 \%$ & $52 \%$ \\
\hline BMI & $0.59(0.44-0.73)$ & 29.5 & $60 \%$ & $60 \%$ \\
\hline WHR & $0.47(0.29-0.65)$ & 0.85 & $47 \%$ & $38 \%$ \\
\hline \multicolumn{5}{|c|}{ Total (combined males and females) } \\
\hline $\mathrm{HC}$ & $0.64(0.58-0.69)$ & 95.5 & $78 \%$ & $42 \%$ \\
\hline WC & $0.63(0.57-0.69)$ & 84.5 & $78 \%$ & $43 \%$ \\
\hline WHtR & $0.62(0.56-0.68)$ & 0.5 & $71 \%$ & $47 \%$ \\
\hline BMI & $0.59(0.53-0.65)$ & 25.3 & $67 \%$ & $46 \%$ \\
\hline WHR & $0.53(0.47-0.60)$ & 0.89 & $60 \%$ & $41 \%$ \\
\hline
\end{tabular}

Comparing all the five anthropometric indices against the three outcome variables of FBS, prediabetes, and diabetes using a simple scoring system (Table 6), the best predictor of FBS, prediabetes, and diabetes (combined) was WC and the predictive ability was greater in men than in women. The next best predictor was WHtR which had greater predictive ability in women than in men. Therefore, WC and WHtR were the overall best anthropometric predictors of FBS, diabetes and prediabetes in both sexes at cut-off values of $87 \mathrm{~cm}$ and 0.5 respectively.

\section{Discussion}

The prevalence rates of prediabetes and diabetes among bank employees in this study were $7 \%$ and $4 \%$, respectively. The prevalence of both prediabetes and diabetes was higher in men $(3.9 \%$ and $7.5 \%)$ than 


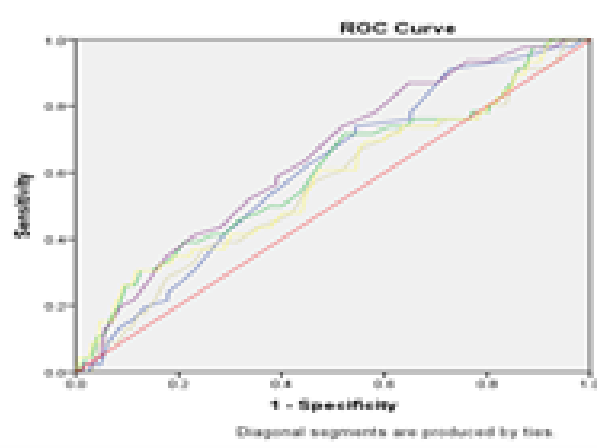

1. Diabetes

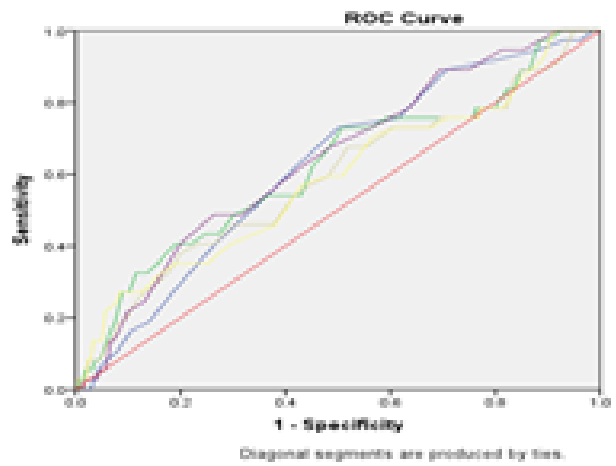

3. Diabetes (Men)

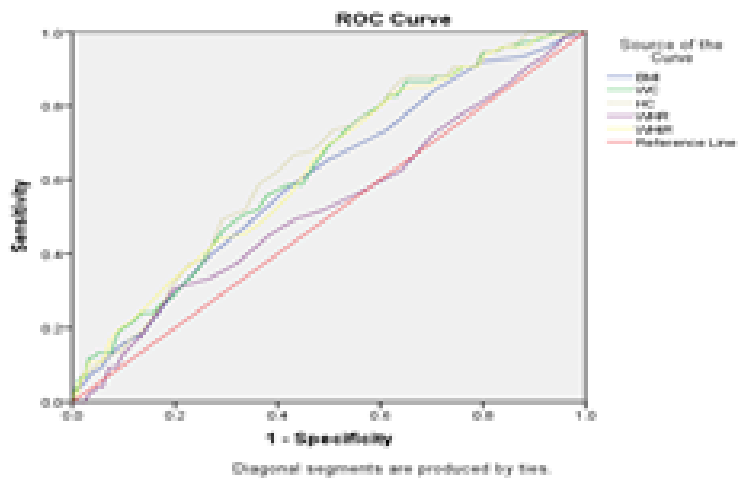

5. Prediabetes (Men)

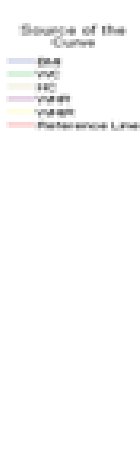

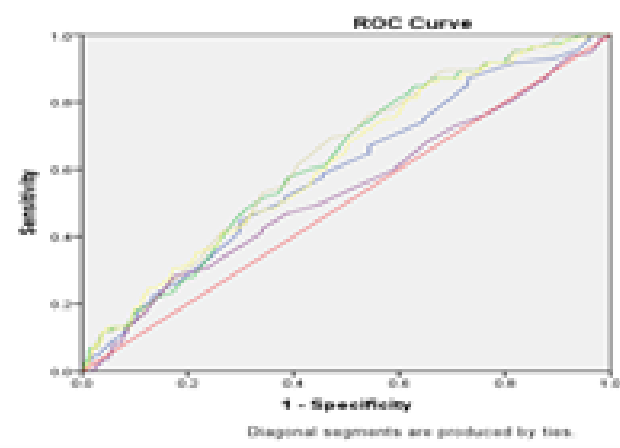

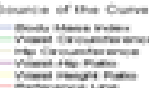

2. Prediabetes
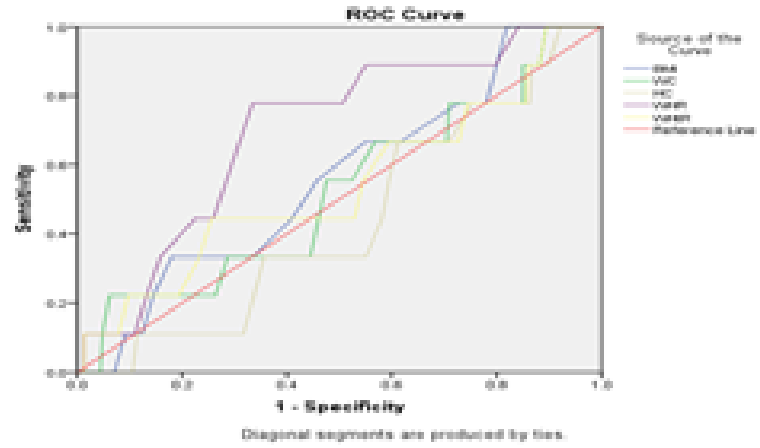

4. Diabetes (Women)

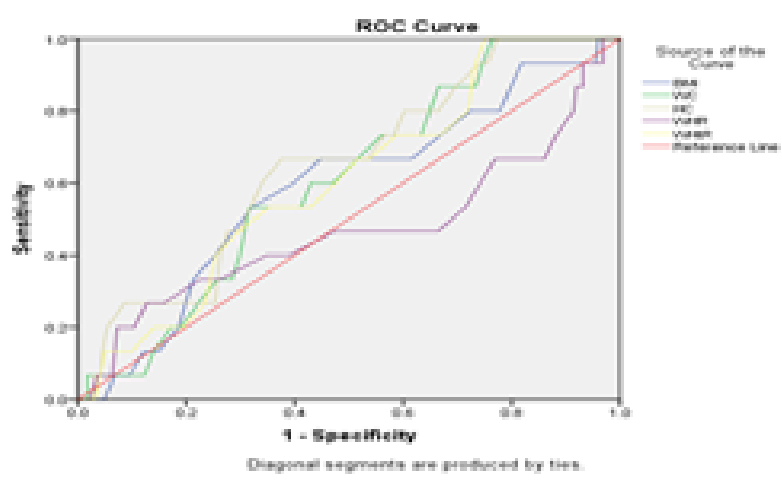

6. Prediabetes (Women)

Figure 1. AUC for the various anthropometric predictors of diabetes and prediabetes 
Table 6. Comparison and scoring system on the predictive ability of anthropometric indices

\begin{tabular}{|c|c|c|c|c|}
\hline & FBS & Prediabetes & Diabetes & Total score \\
\hline \multicolumn{5}{|c|}{ Both } \\
\hline WC & 5 & 4 & 4 & 13 \\
\hline WHtR & 4 & 3 & 2 & 9 \\
\hline $\mathrm{HC}$ & 3 & 5 & 1 & 9 \\
\hline BMI & 2 & 2 & 3 & 7 \\
\hline WHR & 1 & 1 & 5 & 7 \\
\hline \multicolumn{5}{|c|}{ Men } \\
\hline WC & 5 & 4 & 4 & 13 \\
\hline WHtR & 4 & 3 & 2 & 9 \\
\hline $\mathrm{HC}$ & 3 & 5 & 1 & 9 \\
\hline BMI & 2 & 2 & 3 & 7 \\
\hline WHR & 1 & 1 & 5 & 7 \\
\hline \multicolumn{5}{|c|}{ Women } \\
\hline $\mathrm{WC}$ & 5 & 4 & 2 & 11 \\
\hline WHtR & 4 & 3 & 3 & 10 \\
\hline $\mathrm{HC}$ & 3 & 5 & 1 & 9 \\
\hline BMI & 2 & 2 & 4 & 8 \\
\hline WHR & 1 & 1 & 5 & 7 \\
\hline
\end{tabular}

in women $(3.8 \%$ and $7 \%)$. This prevalence of diabetes $(4 \%)$ is much lower than the rate reported by Gezawa et al. [11] in Maiduguri, northeast Nigeria (7\%) and that reported by Hajian-Tilaki and Heidari [4] in in Babol, northern Iran (14\%). However, the higher prevalence of diabetes in men than in women is consistent with both studies $[4,11]$.

Similarly, the statistically significant associations between the anthropometric indexes and FBS have been clearly demonstrated in previous studies $[1,2,5,11,13]$. The finding of insignificant correlation between BMI $(\mathrm{r}=0.101, \mathrm{p}=0.121)$ and WHR $(\mathrm{r}=0.032, \mathrm{p}=0.621)$ with FBS in women sharply contradicts the significant relationship (BMI, $\mathrm{r}=0.399, \mathrm{p}<0.01$ ) reported by Farah et al. [14] in Andhra Pradesh, south-eastern India, and this phenomenon needs further exploration in future studies.

The finding of $\mathrm{WC}(\mathrm{r}=0.231, \mathrm{p}<0.0001)$ as the best predictor of FBS in both sexes also contradicts findings from two reviewed studies: one in Nigeria and the other in India [11,14]. Both reviewed studies were conducted on youths and reported BMI as the best predictor of FBS using the same methodology. However, the Indian study had WC as the next best predictor of FBS $(r=0.293, p<0.05)$ after BMI $(r=0.399$, $\mathrm{p}<0.01)$ [14]. A smaller sample, limited geographical cover and the recruitment of young adults in the comparison studies may probably explain this disparity $[11,14]$.

This study also found $\mathrm{HC}$ as the best predictor of prediabetes followed by WC and WHtR with BMI and WHR as the worst predictor. The cut-off values for $\mathrm{HC}$ were $96.5 \mathrm{~cm}$ in men and $107.5 \mathrm{~cm}$ in women; WC $85.5 \mathrm{~cm}$ (men) and $88.5 \mathrm{~cm}$ (women); and WHtR 0.5 (men) and 0.55 (women). No previous study explored the predictive value of $\mathrm{HC}$ in prediabetes. Studies in this respect focused mainly on WC, WHR, and BMI.

Haghighatdoost et al. in Iran found BMI and WC as insignificant predictors of prediabetes $(\mathrm{p}>0.05)$ with WC having a higher cut-off value of $89.7 \mathrm{~cm}$ in men and comparable value of $88.2 \mathrm{~cm}$ in women [6]. In contrast, Pandey et al. study found WC as a significant predictor of prediabetes in Indian children with boys having a higher AUC and cutoff values $(0.804 ; 0.795)$ than girls $(82.5 \mathrm{~cm} ; 80.3 \mathrm{~cm})$, respectively [15]. The lower cut-off values can be explained by age since the participants in the Indian study did not attain full adult size.
The best anthropometric predictor of diabetes in both sexes was WHR with identical cut-off values (0.90) but higher AUC in women (0.70) than in men (0.64). This finding differs from those reported by two independent Iranian studies with conflicting results. Hajian-Tilaki and Heidari reported WHtR at cut-off value of 0.51 for both sexes as the best predictor of diabetes, while Haghighatdoost et al. found BMI at cut-off value of $28.5 \mathrm{~kg} / \mathrm{m}^{2}$ [4,6]. A third national study in neighboring Cameroun found $\mathrm{WC}$ as the best predictor of diabetes [7]. Interestingly, WC was the next best predictor of diabetes in this study as well as in the two quoted Iranian studies, but the cut-off values vary exponentially from $85.5 \mathrm{~cm}$ to $98.5 \mathrm{~cm}$. This wide difference may be accounted for by racial and ethnic variability between Africans and Iranians.

Using an arbitrary scoring system, the best anthropometric predictor of all the three outcomes combined (FBS, prediabetes and diabetes) in both sexes was WC followed by WHtR and the predictive ability of these indices was greater in men than in women.

Obesity, especially central obesity, is a major risk factor for both prediabetes and diabetes $[3,7,8]$. As the prevalence of obesity increases, that of prediabetes and diabetes is predicted to rise reciprocally $[16,17]$. Therefore, measures like WC, which estimate abdominal obesity are better predictors of diabetes than BMI which is a marker of general obesity. Measures of abdominal obesity such as WC, WHtR and WHR correlate well with regional fat distribution $[1,2,16,18]$.

\section{Conclusion}

In both sexes, the best anthropometric predictors of FBS, prediabetes, and diabetes were WC, HC, and WHR respectively. The emerging predictive value of $\mathrm{HC}$ in prediabetes needs to be further researched. The overall best predictor of FBS, prediabetes, and diabetes combined was WC, followed by WHtR.

WC and WHtR are measures of visceral and abdominal obesity. The findings in this study suggest that WC and WHtR can predict FBS, prediabetes, and diabetes better than the other anthropometric indices of obesity. WC and WHtR at cut-off values of $87 \mathrm{~cm}$ and 0.5 respectively, are appropriate discriminative anthropometric measures for both prediabetes and diabetes in both sexes.

Since both truncal obesity and abnormal blood sugar are risk factors for cardiovascular disease, reducing WC can potentially prevent 
diabetes in high-risk populations or improve glycemic control and cardiovascular outcomes in diabetics.

\section{Acknowledgements}

The authors thank all those who participated in the collection of the primary data and the management of the bank for giving us the permission to use the dataset.

\section{Conflict of interest}

The authors declare that they have no conflict of interest.

\section{References}

1. Bray GA, Kim KK, Wilding JP, World Obesity Federation (2017) Obesity: a chronic relapsing progressive disease process. A position statement of the World Obesity Federation. Obesity Reviews 18: 715-723. [Crossref]

2. Moore KJ, Shah R (2020) Introduction to the obesity, metabolic syndrome, and CVD compendium. Circulation Research 126: 1475-1476.

3. Zhou C, Zhan L, Yuan J, Tong X, Peng Y (2019) Comparison of visceral, general and central obesity indices in the prediction of metabolic syndrome in maintenance hemodialysis patients. Eating and Weight Disorders-Studies on Anorexia, Bulimia and Obesity 9: 1-8. [Crossref]

4. Hajian-Tilaki K, Heidari B (2015) Is waist circumference a better predictor of diabetes than body mass index or waist-to-height ratio in Iranian adults? International Journal of Preventive Medicine 1: 5 .

5. Sebo P, Beer-Borst S, Haller DM, Bovier PA (2008) Reliability of doctors' anthropometric measurements to detect obesity. Preventive Medicine 47: 389-393.

6. Haghighatdoost F, Amini M, Feizi A, Iraj B (2017) Are body mass index and waist circumference significant predictors of diabetes and prediabetes risk: Results from population based cohort study. World Journal of Diabetes 8: 365 .

7. Mbanya VN, Kengne AP, Mbanya JC, Akhtar H (2015) Body mass index, wais circumference, hip circumference, waist-hip-ratio and waist-height-ratio: Which is the better discriminator of prevalent screen-detected diabetes in a Cameroonian population? Diabetes Research and Clinical Practice 108: 23-30.
8. Oguoma VM (2016) Anthropometric indices: How they compare in screening of cardio-metabolic risks in a Nigerian sub-population. African Journal of Medicine and Medical Sciences 45: 91-98.

9. Oli JM, Adeyemo AA, Okafor GO, Ofoegbu EN, Onyenekwe B (2009) Basal insulin resistance and secretion in Nigerians with type 2 diabetes mellitus. Metabolic Syndrome and Related Disorders 1: 595-600.

10. Akinola OB, Omotoso OG, Akinlolu AA, Ayangbemi KD (2014) Identification of the anthropometric index that best correlates with fasting blood glucose and BMI in postpubescent female Nigerians. Anatomy Journal of Africa 3: 324-348.

11. Gezawa ID, Puepet FH, Mubi BM, Uloko AE, Bakki B, et al. (2015) Sociodemographic and anthropometric risk factors for type 2 diabetes in Maiduguri, NorthEastern Nigeria. Sahel Medical Journal 18: 1.

12. Yakubu IM, Bigelow P (2019) Prevalence and determinants of overweight, obesity, prehypertension, and hypertension in the banking industry in Nigeria: An analysis of a nationwide dataset. Texila International Journal of Public Health 7.

13. Ho SC, Chen YM, Woo JL, Leung SS, Lam TH (2001) Association between simple anthropometric indices and cardiovascular risk factors. International Journal of Obesity 25: 1689-1697.

14. Farah N, Devi MS, Patnaik AK (2015) Correlation of adiposity with fasting blood glucose in young individuals. Scholars J Appl Med Sci 3: 1534153-8.

15. Pandey U, Midha T, Rao YK, Katiyar P, Wal P, et al. (2017) Anthropometric indicators as predictor of pre-diabetes in Indian adolescents. Indian Heart Journal 69: 474-479.

16. Hall JE, do Carmo JM, da Silva AA, Wang Z, Hall ME (2015) Obesity-induced hypertension: interaction of neurohumoral and renal mechanisms. Circulation Research 13: $991-1006$

17. Narkiewicz K (2006) Obesity and hypertension-the issue is more complex than we thought. Nephrology Dialysis Transplantation 1: 264-267.

18. Feller S, Boeing H, Pischon T (2010) Body mass index, waist circumference, and the risk of type 2 diabetes mellitus: implications for routine clinical practice. Deutsches Ärzteblatt International 107: 470. [Crossref]

Copyright: (C2020 Yakubu IM. This is an open-access article distributed under the terms of the Creative Commons Attribution License, which permits unrestricted use, distribution, and reproduction in any medium, provided the original author and source are credited. 